\title{
L-Citrulline Level in Pregnant Adolescent With Severe Preeclampsia Compared to Without Preeclampsia: A Cross-sectional Study
}

\author{
Dini Hidayat $^{*}$, Tri Mulya Fitriasari, Tono Djuwantono, Yudi Mulyana Hidayat, Setyorini Irianti, \\ Mulyanusa A Ritonga, Sunardi Sunardi
}

\begin{abstract}
Objectives: Numerous studies have been performed on the effect of L-arginine supplementation on improving maternal outcomes, but studies on the impact of L-citrulline in pregnancy are limited. This study aimed to evaluate the L-citrulline level in pregnant adolescents with severe preeclampsia compared to those without preeclampsia.

Materials and Methods: This is a cross-sectional study among 60 adolescent pregnant women giving birth in the Emergency Room and Delivery Room in Hasan Sadikin Hospital, Bandung, Indonesia from May to October 2020. The L-citrulline levels were examined in women with severe preeclampsia $(n=30)$ and those without preeclampsia $(n=30)$.

Results: The L-citrulline level in adolescent pregnancies with severe preeclampsia was lower than those without preeclampsia $(79.7 \pm 40.4,122.3 \pm 61.9 \mathrm{pg} / \mathrm{mL}$, respectively and $P=0.003)$. But no correlations were observed between L-citrulline levels with systolic blood pressure, diastolic blood pressure, and mean arterial pressure (MAP).

Conclusions: There was a difference in serum L-citrulline level of adolescent pregnancies with severe preeclampsia and without pre-eclampsia.

Keywords: Severe preeclampsia, L-citrulline, Nitric oxide
\end{abstract}

\section{Introduction}

Preeclampsia is a common complication in pregnant mother characterized by hypertension and new-onset proteinuria at gestational age above 20 weeks (1-4). From January 2009 to December 2013, there are as many as 8275 childbirths in Hasan Sadikin Hospital, Bandung, Indonesia. The total number of preeclampsia and eclampsia cases was 1811 . The total maternal mortality during this period was 106 cases, with 61 cases $(57.5 \%)$ of which were preeclampsia and eclampsia (5). One of the risk factors known for preeclampsia is too old or too young pregnant mothers (adolescent pregnancy). Adolescent pregnancy increases the risk of adverse pregnancy outcomes such as abortion, anemia, urinary tract infections, premature rupture of the membrane, stillbirth, premature labor, etc $(6,7)$.

Preeclampsia in adolescent pregnancies occurs almost twice as much as in adulthood (8). The pathogenesis of preeclampsia is multifactorial and involves angiogenic factors such as nitric oxide (NO) soluble endoglin, placental growth factor, vascular endothelial growth factor (VEGF), and soluble receptors from VEGF such as soluble fms-like tyrosine kinase-1 (sFlt-1) (9-11).

One of the most studied substances related to preeclampsia is NO. NO is a substance produced from
L-arginine conversion by the NO synthase (NOS) enzyme. In severe pre-eclampsia, less $\mathrm{NO}$ is produced. The presence of $\mathrm{NO}$ production disorders in pregnancy causes vasodilatation dysfunction, which causes an increase in blood pressure (12-15). Research on L-citrulline levels is important in adolescents, as one of the causes of high pregnancy complications in adolescents is related to nutrition. Several studies show that malnutrition is a contributor factor to maternal mortality and morbidity worldwide (16-18). As far as our knowledge, research on L-citrulline in pregnancy is still limited

This study aimed to determine the differences in L-citrulline levels in adolescent pregnancies with severe preeclampsia and without preeclampsia. If lower and statistically significant L-citrulline levels are found, then L-citrulline supplementation can be considered to prevent severe preeclampsia.

\section{Materials and Methods}

Study Design and Participants

In this cross-sectional study, 60 adolescent pregnant women referred to the obstetrics clinic or emergency ward of Dr. Hasan Sadikin Hospital, Bandung, Indonesia, for delivery from May to October 2020 were evaluated in two groups ( $n=30 /$ each): the case (severe preeclampsia) and 
Key Messages

- L-citrulline is a metabolite that plays an important role in the pathogenesis of preeclampsia. The lower the L-citrulline levels, the more frequent severe preeclampsia tends to develop. Thus, L-citrulline supplementation can be considered for the prevention of severe preeclampsia.

control (normal pregnancy).

The inclusion criteria for the case group were adolescent pregnant women (aged 10-19 years), gestational age $>20$ weeks, severe preeclampsia diagnosis based on physical examinations and laboratory tests. All participants with incomplete medical records, systemiclupus erythematosus, stroke, deep vein thrombosis, abnormal serum urea, creatinine, serum glutamic oxaloacetic transaminase (GOT), serum glutamic pyruvic transaminase (SGPT), and lactate dehydrogenase (LDH) were excluded. The control group was chosen from adolescent pregnant women presented to obstetrics clinic or emergency room without preeclampsia diagnosis.

\section{Data Sources/Measurement}

A $5 \mathrm{~mL}$ of venous blood samples were taken from all participants in two groups and put into an ethylenediaminetetraacetic acid (EDTA) or heparin anticoagulant tubes for L-citrulline level assay. Then, the samples were centrifuged for 15 mins at $1000 \times \mathrm{g}$ at 2-8 minutes within 30 minutes after retrieval. Assay were carried out using ELISA method with competitive inhibition enzyme immunoassay technique (CEA505Ge 96 Tests Enzyme-linked Immunosorbent Assay Kit for Citrulline (Cit), Cloud-Clone Corp., USA) (19). A monoclonal antibody specific to citrulline has been pre-coated onto a microplate. A competitive inhibition reaction is launched between biotin labeled citrulline and unlabeled citrulline (Standards or samples) with the precoated antibody specific to citrulline. After incubation the unbound conjugate is washed off. Next, avidin conjugated to horseradish peroxidase is added to each microplate well and incubated. The amount of bound horseradish peroxidase conjugate is reverse proportional to the concentration of citrulline in the sample. After addition of the substrate solution, the intensity of color developed is reverse proportional to the concentration of citrulline in the sample.

\section{Variables}

After recruiting the subjects, the L-citrulline level were compared between the case and control group and the correlation with the blood pressure were evaluated. Preeclampsia was defined as elevated blood pressure (more than 140/100 mm Hg) after 20 weeks of gestation and proteinuria $(\geq+1$ in dipstick urine sample or $\geq 300$ mg during 24 hours or urine protein: creatinine ratio $\geq$ $0.3)$. The maternal age, gestational age, body mass index
(BMI), systolic blood pressure, diastolic blood pressure, mean arterial pressure (MAP), and L-citrulline level were compared between the case and control group as studied variables. The maternal age was defined as the age of subjects when presented to the hospital. The gestational age was calculated based on the first day of the last menstrual period. The BMI of subjects were calculated based on the BMI formula (weight in kilograms divided by height in meters squared). The systolic blood pressure was the pressure of the blood exerting against artery walls when the heart beats, detected as the first beat sound heard with stethoscope. The diastolic blood pressure was the pressure of blood is exerting against the artery walls while the heart is resting between beats, detected as the last beat sound heard with stethoscope. The MAP was calculated with the formula of systolic blood pressure $+(2$ $\mathrm{X}$ diastolic blood pressure)]/3. While the L-citrulline level was measured with ELISA method.

\section{Sample Size}

Given the formula of sample size calculation in numeric and categorical analytical studies with unpaired design, (N $=2 \mathrm{~S} 2(\mathrm{Z} \alpha+\mathrm{Z} \beta) 2 /(\overline{\mathrm{X}} 1-\overline{\mathrm{X}} 2) 2, \mathrm{Z} \alpha$ and $\mathrm{Z} \beta$ were considered 1.96 and 1.64 , respectively, $\alpha=5 \%$, and power $95 \%$. The $\bar{X} 1, \bar{X} 2$, and $S$ were $44.1 \pm 4.1,28.6 \pm 1.2$, and 11.12, respectively (20). With a $10 \%$ dropout, the sample size of 15 for each group was calculated (total sample size $=$ 30). Finally, based on the rule of thumb for the minimum numerical comparative test of 30 samples in each group, we considered a sample size of 60 people $(n=30 /$ each group).

\section{Data Analysis}

For the statistical analyses, we use the Shapiro-Wilk test to assessed data normality. To compare the characteristics between both groups, an independent $t$ test was used. The relationship between L-citrulline levels and blood pressure in severe preeclampsia was assessed using Spearman rank correlation. The cut-off point was analyzed using the receiver operating characteristics (ROC) curve. SPSS version 25.0 (SPSS Inc., Chicago, IL, USA) was used in this study and $P$ value of $\leq 0.05$ was considered significant.

\section{Results}

Totally, 30 adolescent mothers with severe preeclampsia and 30 adolescent mothers with normal pregnancy were recurred in this study. There were not significant differences between two groups in terms of maternal age, gestational age, and body mass index (Table 1).

All of the statistical tests in L-citrulline levels show that the difference between both groups is not significant $(P=0.312, \quad P=0.081$, respectively). The L-citrulline average level among adolescent pregnancies with severe preeclampsia was $79.7 \pm 40.4 \mathrm{pg} / \mathrm{mL}$, which was lower than in non-preeclampsia adolescent pregnancies of 122.3 $\pm 61.9 \mathrm{pg} / \mathrm{mL}$. These levels had a statistically significant 
Table 1. The Demographic Characteristics of Participants in Two Study Groups

\begin{tabular}{|c|c|c|c|}
\hline Characteristics & Severe Preeclampsia $(n=30)$ & Non-Preeclampsia $(\mathrm{n}=\mathbf{3 0})$ & $P$ Value \\
\hline Age $(y)^{\mathrm{a}}$ & $19(14-19)$ & $18(14-19)$ & 0.901 \\
\hline \multicolumn{4}{|c|}{ Gestational age $(w k)^{b}$} \\
\hline$\leq 34$ & $9(30.0)$ & $16(53.3)$ & 0.067 \\
\hline$>34$ & $21(70.0)$ & $14(46.7)$ & \\
\hline $\mathrm{BMI}\left(\mathrm{kg} / \mathrm{m}^{2}\right)^{*}$ & $23.1(16.7-41.0)$ & $21.8(20.0-25.0)$ & 0.165 \\
\hline
\end{tabular}

BMI: body mass index.

${ }^{a}$ Data presented as Median (Min-Max); Mann Whitney test.

${ }^{\mathrm{b}}$ Data presented as $\mathrm{n}(\%)$; chi-square test.

difference $(P=0.003)$.

Figure 1 shows that the median line and boxplot L-citrulline levels are not parallel between adolescent pregnancies with severe preeclampsia and without preeclampsia, which shows a significant difference. We conducted a statistical correlation analysis between L-citrulline levels and blood pressure assessed from systolic blood pressure, diastolic blood pressure, and MAP. Therefore, there were no correlations between L-citrulline levels and systolic and diastolic blood pressure (Table 2).

\section{Discussion}

From the characteristics of subjects in this study, we found that the mean age of participants with severe preeclampsia and normal pregnancy did not show significant differences. In the severe preeclampsia group, we found that the mean maternal age was 19 years. This is different

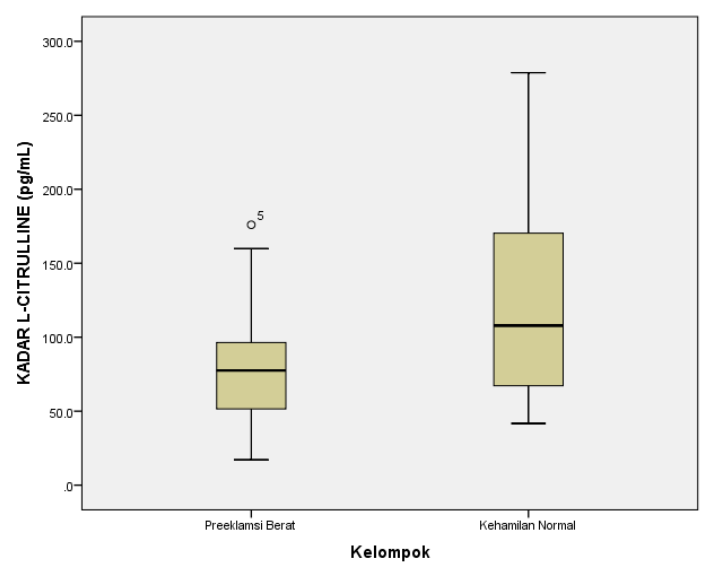

Figure 1. Boxplot Comparison of L-citrulline Levels in Adolescent Pregnancy with Severe Preeclampsia and Non-preeclampsia Adolescent Pregnancy. from the previous study which found that the highest rate of severe preeclampsia occurred in the 15-17 years old age group (21). However, based on the study, 19 years old belongs to the second-highest group of preeclampsia risk $(8,21,22)$.

The average gestational age in the severe preeclampsia group was more than 34 weeks of gestation. The theory states that maternal factors because delayed preeclampsia compared to early severe preeclampsia, which is mainly caused by placental factors. This condition is different from the literature, which stated that placentation disorder is the main cause of preeclampsia in adolescents $(23,24)$.

In the group of severe preeclampsia women, the average BMI was $23.1 \mathrm{~kg} / \mathrm{m}^{2}$, which is categorized as overweight. The average BMI in the group without preeclampsia was $21.8 \mathrm{~kg} / \mathrm{m}^{2}$, which is categorized as normal. Obesity is associated with a threefold risk of preeclampsia. Obesity is said to increase the occurrence of both early-onset and slow-onset preeclampsia. Obesity causes an increase in the occurrence of inflammation, insulin resistance, and oxidative stress. This causes a decrease in NO levels caused by oxidative stress (25-27).

L-citrulline is a stoichiometric metabolite that results from L-arginine conversion into NO. L-citrulline measurements are probably the most specific and reliable metabolite to assess NOS activity in vivo $(16,17,28)$. In this study, the average of L-citrulline levels in adolescent pregnancy with severe preeclampsia was lower than in non-preeclampsia adolescent pregnancies. This is similar to previous research, which showed that the average serum NO levels of preeclampsia women were lower than normal pregnancies (14). In contrast, another study found that L-citrulline levels in preeclampsia women were higher than in normal women (18). Therefore, further research

Table 2. Correlation of Blood Pressure, and MAP with L-Citrulline Levels

\begin{tabular}{|c|c|c|c|c|c|}
\hline \multirow{2}{*}{ Variables } & \multicolumn{3}{|c|}{ L-citrulline level $(\mathrm{pg} / \mathrm{mL})^{*}$} & \multirow{2}{*}{ Mean \pm SD } & \multirow{2}{*}{ Median (Min-Max) } \\
\hline & $\mathbf{r}$ & $P$ & $\mathbf{R}^{2}$ & & \\
\hline Systolic blood pressure (mm Hg) & 0.072 & 0.352 & 0.005 & $161 \pm 7$ & $160(150-180)$ \\
\hline Diastolic blood pressure (mm Hg) & -0.208 & 0.135 & 0.043 & $105 \pm 5$ & $105(100-110)$ \\
\hline Mean arterial pressure & 0.143 & 0.158 & 0.040 & $123.78 \pm 3.69$ & $123.33(120.00-133.33)$ \\
\hline
\end{tabular}

a Spearman rank correlation.

r: Spearman's rank-correlation coefficient; $R^{2}$ : Spearman's rank-correlation coefficient squared (coefficient of determination). 
is needed with more samples regarding L-citrulline levels.

In this study, the average systolic blood pressure, diastolic blood pressure, and MAP were $161 \mathrm{~mm} \mathrm{Hg}, 105 \mathrm{~mm} \mathrm{Hg}$, and $123.78 \mathrm{~mm} \mathrm{Hg}$, respectively. Statistical correlation test between the blood pressure and L-citrulline levels indicates that there is no correlation between L-citrulline levels and these three parameters $(P>0.005)$. The results in this study are similar to a previous study conducted in 36 heavily preeclampsia women (29). The study found that L-citrulline levels did not correlate with systolic blood pressure, diastolic blood pressure, and MAP. This suggests that a lack of production of $\mathrm{NO}$ did not cause the increase in blood pressure. However, further research is still needed regarding the relationship between NOS genes, NOS levels, L-citrulline levels, and blood pressure. From a previous research which administered L-arginine supplements to women with gestational hypertension compared to those given placebo showed a difference in blood pressure reduction (30). Similarly, research conducted in 2013 showed that the administration of L-arginine supplementation in cases of hypertension in pregnancy showed a significant effect in lowering diastolic blood pressure compared to placebo administration (31).

Theoretically, this study differs from the hypothesis that L-citrulline levels, which reflect the NO levels, were correlated with blood pressure. NO is related to endothelial regulation, blood pressure regulation, and cardiovascular balance. Endothelial dysfunction, which is characterized by NO bioavailability disorders, is regarded as one of the risk factors for hypertension and cardiovascular disease. NO causes vascular relaxation mediated by the effects of 3', 5'-cyclic guanosine monophosphate, which produces vasodilation of smooth muscle cells, prevention of adhesion and platelet aggregation, and has antimigration effects of leukocytes, endothelial cells, as well as smooth muscles, thus preventing the occurrence of atherosclerosis. Hypertensive conditions caused by increased reactive oxygen species reduce the bioavailability of NO. The bioavailability of NO can be improved by pharmacological and nonpharmacological methods. One of the pharmacological methods is the administration of L-arginine. Research conducted on animals and women shows the presence of beneficial effects $(29,32)$.

\section{Limitations of the Study}

In this study, no dietary recall was conducted for foods containing L-arginine which can affect L-citrulline levels of the research subjects and no examination of other factors such as NO Synthase level which theoretically affects the metabolic process of NO to L-citrulline.

\section{Conclusions}

L-citrulline level in the adolescent mothers with severe preeclampsia was significantly lower than non-preeclampsia adolescent mothers. There are no correlations between L-citrulline levels and systolic blood pressure, diastolic and MAP in severe preeclampsia women.

\section{Authors' Contribution}

$\mathrm{DH}, \mathrm{TM}, \mathrm{TD}, \mathrm{YM}$, and $\mathrm{S}$ designed the study and conducted the research. $\mathrm{SI}$ and $\mathrm{MH}$ monitored, evaluated, and analyzed the result of the study. Further, TM, TD, YM, DH, SI, and MH reviewed the article. All authors approved the final manuscript and take responsibility for the integrity of the data.

\section{Conflict of Interests}

Authors declare that they have no conflict of interests.

\section{Ethical Issues}

Health Research Ethics Committee of Hasan Sadikin Hospital, Bandung, Indonesia approved this research protocol (Code: LB.02.01/X.6.5/113/2020). Written informed consent were obtained from all participants before initiating the study. Subject confidentiality was maintained during and after the study.

\section{Financial Support}

This research is funded by the authors themselves. We received no financial support from other external sources.

\section{References}

1. Kirbas A, Ersoy AO, Daglar K, et al. Prediction of preeclampsia by first trimester combined test and simple complete blood count parameters. J Clin Diagn Res. 2015;9(11):QC20-23. doi:10.7860/ jcdr/2015/15397.6833

2. Serin S, Avcı F, Ercan O, Köstü B, Bakacak M, Kıran H. Is neutrophil/ lymphocyte ratio a useful marker to predict the severity of preeclampsia? Pregnancy Hypertens. 2016;6(1):22-25. doi:10.1016/j. preghy.2016.01.005

3. Henderson JT, Thompson JH, Burda BU, Cantor A. Preeclampsia screening: evidence report and systematic review for the U.S. Preventive Services Task Force. JAMA. 2017;317(16):1668-1683. doi:10.1001/jama.2016.18315

4. Rana S, Lemoine E, Granger JP, Karumanchi SA. Preeclampsia: pathophysiology, challenges, and perspectives. Circ Res. 2019;124(7):1094-1112. doi:10.1161/circresaha.118.313276

5. Burhanuddin SM, Krisnadi SR, Pusianawati D. Patients Characteristics and Outcomes on Early Onset and Late Onset Preeclampsia at Dr Hasan Sadikin Hospital Bandung. Indones J Obstet Gynecol Sci. 2018;1(2):117-124. doi:10.24198/obgynia. v1n2.12

6. Darroch JE, Woog V, Bankole A, Ashford LS. Costs and Benefits of Meeting the Contraceptive Needs of Adolescents. Guttmacher Institute; 2016.

7. Sama CB, Ngasa SN, Dzekem BS, Choukem SP. Prevalence, predictors and adverse outcomes of adolescent pregnancy in sub-Saharan Africa: a protocol of a systematic review. Syst Rev. 2017;6(1):247. doi:10.1186/s13643-017-0650-0

8. Kawakita T, Wilson K, Grantz KL, Landy HJ, Huang CC, GomezLobo V. Adverse maternal and neonatal outcomes in adolescent pregnancy. J Pediatr Adolesc Gynecol. 2016;29(2):130-136. doi:10.1016/j.jpag.2015.08.006

9. Chaiworapongsa T, Chaemsaithong P, Yeo L, Romero R. Preeclampsia part 1: current understanding of its pathophysiology. Nat Rev Nephrol. 2014;10(8):466-480. doi:10.1038/nrneph.2014.102

10. Chaiworapongsa T, Chaemsaithong P, Korzeniewski SJ, Yeo L, Romero R. Pre-eclampsia part 2: prediction, prevention and management. Nat Rev Nephrol. 2014;10(9):531-540. doi:10.1038/ nrneph.2014.103

11. Karumanchi SA. Angiogenic factors in preeclampsia: from diagnosis to therapy. Hypertension. 2016;67(6):1072-1079. doi:10.1161/hypertensionaha.116.06421

12. Penatti BS, Battistella MDB, da Silva MT, et al. 95 Nitric oxide in preeclampsia: clinical correlations and serum levels: biomarkers, prediction of preeclampsia. Pregnancy Hypertens. 2016;6(3):224- 
225. doi:10.1016/j.preghy.2016.08.177

13. Adu-Bonsaffoh K, Antwi DA, Obed SA, Gyan B. Nitric oxide dysregulation in the pathogenesis of preeclampsia among Ghanaian women. Integr Blood Press Control. 2015;8:1-6. doi:10.2147/ibpc.s68454

14. Choi JW, Im MW, Pai SH. Nitric oxide production increases during normal pregnancy and decreases in preeclampsia. Ann Clin Lab Sci. 2002;32(3):257-263.

15. Hung $\mathrm{JH}$. Oxidative stress and antioxidants in preeclampsia. J Chin Med Assoc. 2007;70(10):430-432. doi:10.1016/s17264901(08)70032-7

16. Tain YL, Huang LT, Lee CT, Chan JY, Hsu CN. Maternal citrulline supplementation prevents prenatal $\mathrm{N}(\mathrm{G})$-nitro-L-arginine-methyl ester (L-NAME)-induced programmed hypertension in rats. Biol Reprod. 2015;92(1):7. doi:10.1095/biolreprod.114.121384

17. Weckman AM, McDonald CR, Baxter JB, Fawzi WW, Conroy AL, Kain KC. Perspective: L-arginine and L-citrulline supplementation in pregnancy: a potential strategy to improve birth outcomes in low-resource settings. Adv Nutr. 2019;10(5):765-777. doi:10.1093/advances/nmz015

18. Benedetto C, Marozio L, Neri I, Giarola M, Volpe A, Facchinetti F. Increased L-citrulline/L-arginine plasma ratio in severe preeclampsia. Obstet Gynecol. 2000;96(3):395-399. doi:10.1016/ s0029-7844(00)00939-x

19. CEA505Ge 96 Tests Enzyme-linked Immunosorbent Assay Kit for Citrulline (Cit), Organism Species: Pan-Species (General) Instruction Manual. 12th ed: Cloud-Clone Corp.

20. Xu H, Shatenstein B, Luo Z-C, Wei S, Fraser W. Role of nutrition in the risk of preeclampsia. Nutr Rev. 2009;67:639-657. doi: 10.1111/j.1753-4887.2009.00249.x

21. Sheen JJ, Huang Y, Wright JD, Goffman D, D'Alton ME, Friedman AM. 318: Maternal age and preeclampsia outcomes. Am J Obstet Gynecol. 2019;220(1):S222-S223. doi:10.1016/j. ajog.2018.11.339

22. Ganchimeg T, Ota E, Morisaki N, et al. Pregnancy and childbirth outcomes among adolescent mothers: a World Health Organization multicountry study. BJOG. 2014;121 Suppl 1:40-48. doi:10.1111/1471-0528.12630

23. Aziz A, Mose JC. The differences of characteristic, management, maternal and perinatal outcomes among early and late onset preeclampsia. OAlib. 2016;3(6):e2750. doi:10.4236/ oalib. 1102750

24. Sonek J, Krantz D, Carmichael J, et al. First-trimester screening for early and late preeclampsia using maternal characteristics, biomarkers, and estimated placental volume. Am J Obstet Gynecol. 2018;218(1):126.e121-126.e113. doi:10.1016/j. ajog.2017.10.024

25. Dekker G, Sukcharoen N. Etiology of preeclampsia: an update. J Med Assoc Thai. 2004;87 Suppl 3:S96-103.

26. Roberts JM, Bodnar LM, Patrick TE, Powers RW. The role of obesity in preeclampsia. Pregnancy Hypertens. 2011;1(1):6-16. doi:10.1016/j.preghy.2010.10.013

27. Roberts JM. Endothelial dysfunction in preeclampsia. Semin Reprod Endocrinol. 1998;16(1):5-15. doi:10.1055/s-2007-1016248

28. Khalil RA, Granger JP. Vascular mechanisms of increased arterial pressure in preeclampsia: lessons from animal models. Am J Physiol Regul Integr Comp Physiol. 2002;283(1):R29-45. doi:10.1152/ajpregu.00762.2001

29. Karmia HR, Afriwardi A, Ali H, Mose JC, Yusrawati Y. The correlation of L-citrulline levels with blood pressure in severe preeclampsia. Indones Biomed J. 2020;12(1):15-18. doi:10.18585/ inabj.v12i1.964

30. Facchinetti F, Saade GR, Neri I, Pizzi C, Longo M, Volpe A. L-arginine supplementation in patients with gestational hypertension: a pilot study. Hypertens Pregnancy. 2007;26(1):121130. doi:10.1080/10641950601147994

31. Zhu Q, Yue X, Tian QY, et al. Effect of L-arginine supplementation on blood pressure in pregnant women: a meta-analysis of placebocontrolled trials. Hypertens Pregnancy. 2013;32(1):32-41. doi:10. 3109/10641955.2012.697952

32. Hermann M, Flammer A, Lüscher TF. Nitric oxide in hypertension. J Clin Hypertens (Greenwich). 2006;8(12 Suppl 4):17-29. doi:10.1111/j.1524-6175.2006.06032.x

(C) 2022 The Author(s); This is an open-access article distributed under the terms of the Creative Commons Attribution License (http:// creativecommons.org/licenses/by/4.0), which permits unrestricted use, distribution, and reproduction in any medium, provided the original work is properly cited. 